\begin{tabular}{|c|l|}
\hline Title & Polyinstanton axion inflation \\
\hline Author(s) & Kobay ashi, Tatsuo; Uemura, Shohei; Y amamoto, Junji \\
\hline Citation & $\begin{array}{l}\text { Physical review D, 96(2), 026007 } \\
\text { https://doi.org/L0.1103/PhysRevD.96.026007 }\end{array}$ \\
\hline Issue Date & 2017-07-15 \\
\hline Doc URL & http://hdl.handle.net/2115/67108 \\
\hline Rights & ○2017 A merican Physical Society \\
\hline Type & article \\
\hline File Information & PhysRevD96 026007.pdf \\
\hline
\end{tabular}

Instructions for use 


\title{
Polyinstanton axion inflation
}

\author{
Tatsuo Kobayashi, ${ }^{1}$ Shohei Uemura, ${ }^{2}$ and Junji Yamamoto ${ }^{3}$ \\ ${ }^{1}$ Department of Physics, Hokkaido University, Sapporo 060-0810, Japan \\ ${ }^{2}$ Maskawa Institute for Science and Culture, Kyoto Sangyo University, Kyoto 603-8555, Japan \\ ${ }^{3}$ Department of Physics, Kyoto University, Kyoto 606-8502, Japan
}

(Received 19 May 2017; published 12 July 2017)

\begin{abstract}
We investigate the axion inflation model derived by polyinstanton effects in type II superstring theories. Polyinstanton effects are instanton effects corrected by another instanton and they can generate the modulus-axion potential with the double exponential function. Although the axion has a period of small value, this potential can have a flat region because its derivatives are exponentially suppressed by nonperturbative effects. From the view point of the cosmic inflation, such a potential is interesting. In this paper, we numerically study the possibilities of realizing the cosmic inflation. We also study their spectral index and other cosmological observables, numerically.
\end{abstract}

DOI: $10.1103 /$ PhysRevD.96.026007

\section{INTRODUCTION}

The cosmic inflation is an important stage in the history of the Universe. It solves the problems of the standard big bang cosmology such as the flatness problem and horizon problem, and derives the observed fluctuation of the cosmic microwave background. From the viewpoint of underlying theories, such as superstring theory, what the field is deriving the inflation, i.e., inflaton, is one of the key questions to understanding the Universe. It should have sufficiently flat potential. Indeed, various studies have been done within the framework of superstring theory [1].

Axions as well as moduli are good candidates for the inflaton, because axions have continuous shift symmetries. Nonperturbative effects break these continuous shift symmetries to discrete ones, and the potential of the axions can be generated. However, such a potential would be controlled well by discrete shift symmetries. Thus, nonperturbative effects are quite important. Nontrivial backgrounds can also break continuous shift symmetries, generating the axion potential.

The natural inflation [2] and the axion monodromy inflation $[3]^{1}$ are famous realizations of axion inflation models. The simple natural inflation requires a large decay constant, although the typical decay constant would be smaller than the string and Planck scales. A large decay constant can be realized effectively, e.g., by the alignment mechanism [5] and loop effects [6,7]. The simple natural inflation may lead to disfavored predictions on observables, but modulation terms would significantly change predictions on the observables [7-9]. Correction terms in monodromy inflation are also important and they change significantly predictions on the observables $[9,10]$.

String world-sheet instanton corrections are stringycalculable effects. The world-sheet instanton effects can be controlled by modular symmetries, which are characteristic symmetries in string theories. Then, certain modular functions appear as the potential of moduli and axions. The inflation scenario with such modular function terms has also been studied [7,11,12]. Moreover, the mixture of polynomial functions and sinusoidal functions was derived by quantum corrections and studied to realize inflation models [13-15].

Furthermore, one can compute couplings of moduli and axions to the visible sectors, such as gauge bosons, quarks, and leptons, in four-dimensional (4D) low energy effective field theory. Thus, we can explicitly study the thermal history after the axion inflation. For example, we can analyze explicitly the reheating processes after the axion inflation. The axion couplings to the gauge bosons, quarks, and leptons may be rather weak, and the reheating temperature may be rather low $[9,13,15]$. The axions would also appear in phases of couplings among the quarks, leptons, and the Higgs fields. After the inflation, the axion would oscillate, and then coupling phases would vary. Such an oscillation would have important effects on the history of the Universe. For example, the baryon asymmetry may be generated by the oscillation [16].

As mentioned above, various types of the axion inflation models have been studied and several aspects after the inflation have been discussed. It would be interesting to study various possibilities of the axion inflation, and their several aspects. Recently, a new type of nonperturbative effect was studied, that is, the so-called polyinstanton $[17,18]$. That leads to the exponential function of exponential function as the moduli potential term. It would be interesting to study the possibility of the inflation by using such a new type of potential. Indeed, the moduli inflation has been studied by use of such a new potential term [19]. ${ }^{2}$

\footnotetext{
${ }^{2}$ The moduli stabilization has also been studied $[20,21]$.
}

${ }^{1}$ See also [4]. 
(See also [22,23].) The purpose of this paper is to study the possibilities of the axion inflation by use of the new type of axion potential.

This paper is organized as follows. In Sec. II, we show the form of axion potential generated by polyinstanton effects. In Sec. III, we study numerically the polyinstanton axion inflation. Section IV is the conclusion.

\section{POLYINSTANTON POTENTIAL}

The 4D low energy effective field theory of superstring theory is described by supergravity theory, and it includes the dilaton $S$, Kähler moduli $T_{i}$, and complex structure moduli $U_{m}$. Here, we focus on type IIB superstring theory, although our scenario would be applicable to other superstring theories. Hereafter, we use the unit that $M_{\mathrm{Pl}}=1$, where $M_{\mathrm{Pl}}$ denotes the reduced Planck scale, i.e., $M_{\mathrm{Pl}}=2.4 \times 10^{18} \mathrm{GeV}$.

The superpotential $W$ can be written by a combination of perturbative and nonperturbative terms,

$$
W=W_{p}+W_{n p} .
$$

The gaugino condensations and D-brane instanton effects are well-known computable nonperturbative effects in type II superstring theory. The superpotential terms induced by D-brane instantons can be written as [24,25],

$$
W_{n p}=\sum_{i} A_{i}\left(U_{m}\right) e^{-a_{i} \mathcal{S}_{i}}
$$

Here, $\mathcal{S}_{i}$ denotes the action of the D-brane instantons, and it can be obtained as $\mathcal{S}_{i}=\int_{\Gamma_{i}} d V=M S+m_{i, j} T_{j}$, where $\Gamma_{i}$ represents a p-cycle wrapped by the D-brane instanton and $m_{i, j} T_{j}$ represents the Kähler moduli corresponding to the volume of $\Gamma_{i}$. Moduli $T_{i}$ are written as

$$
T_{i}=\tau_{i}+i \phi_{i}
$$

and the real parts $\tau_{i}$ and the imaginary parts $\phi_{i}$ represent the 4-cycle volumes and axions, respectively. In addition, $a_{i}$ is a constant parameter and its value is typically $2 \pi$ for D-brane instantons. In general, $A_{i}\left(U_{m}\right)$ is a function of the complex structure moduli $U_{m}$. Here, we assume that all of the complex structure moduli $U_{m}$ and the dilaton $S$ are stabilized by three-form flux backgrounds [26,27].

On the other hand, the gaugino condensation in the hidden sector $G_{a}$ would generate the following term,

$$
W_{n p}=A_{a} e^{-24 \pi^{2} f_{a} / b_{a}},
$$

where $f_{a}$ and $b_{a}$ are the gauge kinetic function and betafunction coefficient of $G_{a}$, respectively. The real part of the gauge kinetic function provides us with the gauge coupling $g_{a}$, i.e., $1 / g_{a}^{2}=\operatorname{Re}\left(f_{a}\right)$. Including quantum corrections, the gauge kinetic function can be written by

$$
f_{a}=f_{\text {tree }}+f_{1 \text {-loop }}+f_{n p} .
$$

Here, the tree-level gauge kinetic function $f_{\text {tree }}$ is a linear combination of $S$ and $T_{i}$ corresponding to the volume of the cycle which the hidden sector $G_{a}$ wraps. The oneloop correction $f_{1 \text {-loop }}$ is a function of complex structure moduli $U_{m}$ and independent of Kähler moduli $T_{i}$. The nonperturbative correction $f_{n p}$ is given by

$$
f_{n p}=\sum_{j} B_{j}\left(U_{m}\right) e^{-b_{j} T_{j}},
$$

and such a correction can be induced by D-brane instantons having one complex position modulus on the compact space. Thus, in general, we can write nonperturbative terms in the superpotential,

$W_{n p}=\sum_{a} A_{a} \exp \left[-b_{a}\left(\alpha_{a, i} T_{i}+\sum_{j} B_{a, j} e^{-b_{j, k} T_{k}}\right)\right]$,

where we have redefined coefficients including vacuum expectation values of $S$ and $U_{m}$, because we have assumed that the dilaton and complex structure moduli are stabilized. A similar nonperturbative superpotential term would appear through the D-brane instanton terms (2.2) with further nonperturbative corrections. Since it is an instanton correction corrected by other instanton effects, it is called a polyinstanton $[17,18]$. It can generate the potential for the Kähler moduli and stabilize them [19-21].

The $F$-term scalar potential is given by

$$
V_{F}=e^{K}\left[K^{T_{i} \bar{T}_{j}} D_{T_{i}} W D_{\bar{T}_{j}} \bar{W}-3|W|^{2}\right],
$$

where $K$ denotes the Kähler potential and $D_{T_{i}} W=$ $\left(\partial_{T_{i}} K\right) W+\partial_{T_{i}} W$. The Kähler potential is given by $K=$ $-2 \ln \mathcal{V}$ where $\mathcal{V}$ is the volume of the compact space, and the Kähler potential is a function of $T_{i}+\bar{T}_{i}$, i.e., $K\left(T_{i}+\bar{T}_{i}\right)$. In addition, $K^{T_{i} \bar{T}_{j}}$ represents the inverse of $K_{T_{i} \bar{T}_{j}}=\partial_{T_{i}} \partial_{\overline{T_{j}}} K$.

In this paper, we focus on the axion potential generated by the polyinstanton. Suppose that there is a modulus field $T_{0}$, which appears only in the polyinstanton superpotential, but does not appear at the tree-level and single instanton potential, i.e.,

$W=\left.W\left(T_{i}\right)\right|_{i \neq 0}+\left.A_{0} \exp \left[-a\left(T_{i}+C e^{-a_{0} T_{0}}\right)\right]\right|_{i \neq 0}$.

In the limit that $A_{0} \rightarrow 0, \phi_{0}$ remains massless, since $\phi_{0}$ does not appear in the $F$-term scalar potential. However, all the other moduli including $\tau_{0}$ appear in the potential. Here, we assume that these moduli including $\tau_{0}$ are stabilized with heavy masses. ${ }^{3}$ Their masses would be estimated by

\footnotetext{
${ }^{3}$ Recently, the moduli stabilization except axions was explored by loop corrections in [28].
} 
$\left.W\left(\left\langle T_{i}\right\rangle\right)\right|_{i \neq 0}$. Thus, when $\left.A_{0} \ll W\left(\left\langle T_{i}\right\rangle\right)\right|_{i \neq 0}$, the mass of $\phi_{0}$ is much smaller than those of the other moduli fields. Therefore, we integrate all the other fields out, and obtain the superpotential,

$$
W=W_{0}^{\prime}+A_{0}^{\prime} \exp \left[-a C e^{-a_{0} T_{0}}\right],
$$

where $W_{0}^{\prime}=\left.W\left(\left\langle T_{i}\right\rangle\right)\right|_{i \neq 0}$ and $A_{0}^{\prime}=A_{0} \exp \left[-a\left\langle T_{i}\right\rangle\right]$.

The $F$-term scalar potential derived from (2.10) has a very specific form. The second term, $-3 e^{K}|W|^{2}$, is calculated as

$$
\begin{aligned}
-3 e^{K}|W|^{2}= & -3 e^{K}\left(\left|W_{0}^{\prime}\right|^{2}+\bar{W}_{0}^{\prime} A_{0}^{\prime} \exp \left[-a C e^{-a_{0} T_{0}}\right]+\text { H.c. }\right) \\
& +\mathcal{O}\left(A_{0}^{\prime 2}\right),
\end{aligned}
$$

where we have used the approximation, $\left|W_{0}^{\prime}\right| \gg\left|A_{0}^{\prime}\right|$. Similarly, we approximate

$$
\begin{aligned}
e^{K} K^{T_{0}} \bar{T}_{0} & \left|D_{T_{0}} W\right|^{2} \\
\approx & e^{K} K^{T_{0} \bar{T}_{0}}\left\{K_{T_{0}}^{2}\left(\left|W_{0}^{\prime}\right|^{2}+\bar{W}_{0}^{\prime} A_{0}^{\prime} \exp \left[-a C e^{-a_{0} T_{0}}\right]+\text { H.c. }\right)\right. \\
& \left.+K_{T_{0}} \bar{W}_{0}^{\prime} A_{0}^{\prime} a_{0} a C e^{-a_{0} T_{0}} \exp \left[-a C e^{-a_{0} T_{0}}\right]+\text { H.c. }\right\} \\
& +\mathcal{O}\left(A_{0}^{\prime 2}\right) .
\end{aligned}
$$

Then, we obtain the $F$-term scalar potential for the axion generated by a polyinstanton,

$$
\begin{aligned}
V \approx & 2 e^{K}\left|\bar{W}_{0}^{\prime} A_{0}^{\prime}\right| \exp \left[-\delta \cos \left(a_{0} \phi_{0}\right)\right] \\
& \times\left\{\left(K^{T_{0} \bar{T}_{0}} K_{T_{0}}^{2}-3\right) \cos \left[\delta \sin \left(a_{0} \phi_{0}\right)+\theta\right]\right. \\
& \left.+K^{T_{0} \bar{T}_{0}} K_{T_{0}} a_{0} \delta \cos \left[-a_{0} \phi_{0}+\delta \sin \left(a_{0} \phi_{0}\right)+\theta\right]\right\} \\
& + \text { (const) },
\end{aligned}
$$

where $\delta=a C \exp \left[-a_{0}\left\langle\tau_{0}\right\rangle\right]$, and $\theta$ is the phase of the prefactors. We omitted the terms of $\mathcal{O}\left(A_{0}^{\prime 2}\right)$.

In the next section, we study the cosmological inflation using the potential (2.13), which is rather complicated. We estimate that $K^{T_{0} \bar{T}_{0}}, K_{T_{0}}^{2}=\mathcal{O}(1)$. Here, we treat $\delta$ and $a_{0}$ as free parameters from the phenomenological viewpoint. When $a_{0} \delta \gg 1$, the second term in the curly bracket is dominant. Then, the potential for the canonically normalized axion $\phi=\sqrt{2 K_{T_{0} \bar{T}_{0}}} \phi_{0}$ is approximated to

$$
V=A\left(e^{-\delta \cos a \phi} \cos [\delta \sin a \phi-a \phi+\theta]+V_{0}\right) .
$$

Here, we redefined coefficients as $a=a_{0} / \sqrt{2 K_{T_{0} \bar{T}_{0}}}$, and $A=2 e^{K} \bar{W}_{0}^{\prime} A_{0}^{\prime} K^{T_{0} \bar{T}_{0}} a_{0} \delta$. We also added the uplifting term $V_{0}$ to set the potential (local) minimum to be almost $0 .{ }^{4}$ To cancel the former term, $V_{0}$ is of the order

\footnotetext{
${ }^{4}$ Such an uplifting term can be realized by supersymmetry breaking D-brane configuration [27] or spontaneous supersymmetry breaking, i.e., the so-called $F$-term uplifting [29].
}

of $e^{-\delta_{\min }}$, where $\delta_{\min }=\delta \cos a \phi_{\min }$, and $\phi=\phi_{\min }$ is the (local) minimum of the potential (2.14). In the next section, we concentrate on the axion inflation with the above potential.

When $a_{0} \delta=\mathcal{O}(1)$, both terms in the curly bracket of Eq. (2.13) are comparable. In this case, it is difficult to control the potential to be flat. A large $\delta$ in the factor $e^{-\delta}$ is important to make the potential flat. Thus, we do not discuss the case with $\delta=\mathcal{O}(1)$, but concentrate on the case with $\delta \gg 1$.

When $a_{0} \delta \ll 1$, the potential is approximated to

$$
\begin{aligned}
V \sim & A_{1} \cos \theta+V_{0}-\delta\left(A_{1}-A_{2}\right) \cos (a \phi-\theta) \\
& +\frac{1}{2} \delta^{2}\left(A_{1}-2 A_{2}\right) \cos (2 a \phi-\theta)+\mathcal{O}\left(\delta^{3}\right),
\end{aligned}
$$

where $A_{1}$ and $A_{2}$ represent the prefactors including Kähler potential and its derivatives. This potential may realize the potential for the natural inflation with modulation terms.

\section{NUMERICAL ANALYSIS}

In this section, we investigate the polyinstanton potential (2.14) numerically. First, we define the slow-roll parameters,

$$
\varepsilon \equiv \frac{1}{2}\left(\frac{V_{\phi}}{V}\right)^{2}, \quad \eta \equiv \frac{V_{\phi \phi}}{V}, \quad \xi \equiv \frac{V_{\phi} \cdot V_{\phi \phi \phi}}{V^{2}} .
$$

These parameters should be much smaller than 1 during the inflation. We evaluate the $e$-folding number,

$$
N_{e}\left(\phi_{*}\right)=\int_{\phi_{\mathrm{end}}}^{\phi_{*}} d \phi \frac{V}{V_{\phi}}
$$

where $\phi_{\text {end }}$ is the end point of inflation. The $e$-folding number is required to be larger than 50 in order to solve the problems of the standard cosmology. Furthermore, the Planck results constrain the power spectrum of curvature perturbation $P_{\xi}$, its spectral index $n_{s}$, and the tensor-toscalar ratio $r[30,31]$,

$P_{\xi}=2.20 \pm 0.10 \times 10^{-9}, \quad n_{s}=0.9655 \pm 0.0062$,

$$
r<0.12 \text {. }
$$

These can be written by use of the slow-roll parameters as

$$
P_{\xi}=\frac{V}{24 \pi^{2} \varepsilon}, \quad n_{s}=1+2 \eta-6 \varepsilon, \quad r=16 \varepsilon .
$$

Table I shows some examples of the parameters and observables, which almost satisfy the above constraints. The overall coefficient $A$ is decided to impose that power spectrum of curvature perturbation $P_{\xi}$ is $2.2 \times 10^{-9}$. We set $a=2 \pi$ in all of the examples in the table. The table also 
TABLE I. Examples of parameters and observables.

\begin{tabular}{lccccccc}
\hline \hline$\delta$ & $\theta$ & $A$ & $n_{s}$ & $r$ & $N_{e}$ & $\alpha_{s}$ & $m_{\phi}^{2}$ \\
\hline 8.0 & 4.13 & $3.29 \times 10^{-16}$ & 0.953 & $6.02 \times 10^{-7}$ & 61.9 & $-3.66 \times 10^{-4}$ \\
9.0 & 3.16 & $3.46 \times 10^{-16}$ & 0.959 & $9.30 \times 10^{-7}$ & 58.6 & $-4.66 \times 10^{-4}$ & $6.70 \times 10^{-11}$ \\
10.0 & 2.11 & $2.18 \times 10^{-16}$ & 0.963 & $7.65 \times 10^{-7}$ & 56.8 & $-4.70 \times 10^{-4}$ \\
11.0 & 7.25 & $1.08 \times 10^{-16}$ & 0.966 & $4.24 \times 10^{-7}$ & 61.7 & $-3.98 \times 10^{-4}$ & $1.26 \times 10^{-10}$ \\
12.0 & 6.28 & $8.89 \times 10^{-17}$ & 0.966 & $4.89 \times 10^{-7}$ & 55.7 & $-4.61 \times 10^{-4}$ & $1.12 \times 10^{-10}$ \\
\hline \hline
\end{tabular}

shows the inflaton mass $m_{\phi}$ and the running of the spectral index $\alpha_{s}$, which is obtained as

$$
\alpha_{s} \equiv \frac{d n_{s}}{d \ln k} \simeq-24 \varepsilon^{2}+16 \varepsilon \eta-2 \xi .
$$

Figures 1 and 2 show the potential forms with $\delta=8.0$ and 9.0, which are shown in Table I. The left panels in the figures show the form of the potential, while the right panels show the slow-roll parameters, $\varepsilon$ and $\eta$. The figures show that there is a flat region in each potential. In order to realize this flatness, the factor $e^{-\delta}$ is important as it will be explained later. The shaded regions correspond to the field excursions during the inflation, i.e., $\phi_{*}$ and $\phi_{\text {end }}$. The inflaton moves from left to right toward the potential local minimum. ${ }^{5}$

Our inflation model corresponds to the so-called smallfield inflation scenario, where the field excursion $\Delta \phi$ during the inflation is small, i.e., $\Delta \phi<1$. By using the Lyth relation [32],

$$
r \sim 10^{-2}(\Delta \phi)^{2}
$$

this implies that $r$ and $\varepsilon$ are smaller than $\mathcal{O}\left(10^{-2}\right)$. In order to derive $n_{s}=0.9655$, we have to realize $\eta \sim-0.01$. The right panels in Figs. 1 and 2 show that $\eta \sim-0.01$ and $\varepsilon$ is very much suppressed.

Now, let us understand the above numerical behaviors of our inflation model by using some approximations. The first derivative of the potential is given by

$$
\begin{aligned}
V_{\phi}= & A \delta a e^{-\delta \cos a \phi}[-\sin (\delta \sin (a \phi)-2 a \phi+\theta) \\
& \left.+\frac{1}{\delta} \sin (\delta \sin (a \phi)-a \phi+\theta)\right] .
\end{aligned}
$$

Since we assume $\delta \gg 1$, it is approximated by

$V_{\phi} \sim A \delta a e^{-\delta \cos a \phi}(-1) \sin (\delta \sin (a \phi)-2 a \phi+\theta)$.

\footnotetext{
${ }^{5}$ Because of the potential form, there are many local minima. The inflaton reaches one of the minima, which may be the global minimum or one of the local ones. At any rate, those minima are a Planck scale away from each other.
}

Similar to that, the second and third derivatives can be approximated as follows:

$$
\begin{aligned}
V_{\phi \phi} & \sim A \delta^{2} a^{2} e^{-\delta \cos a \phi}(-1) \cos (\delta \sin (a \phi)-3 a \phi+\theta), \\
V_{\phi \phi \phi} & \sim A \delta^{3} a^{3} e^{-\delta \cos a \phi}(-1) \sin (\delta \sin (a \phi)-4 a \phi+\theta) .
\end{aligned}
$$

During the inflation, the potential is dominated by $A V_{0}$. On the other hand, the first and second derivatives in a specific region can be much smaller because of the factor $e^{-\delta \cos a \phi}$ when $\delta$ is sufficiently large. Thus, we can realize the flat potential without severe fine-tunings of parameters. In addition, the ratio $\left(V_{\phi}\right)^{2} / V_{\phi \phi}$ is suppressed by $e^{-\delta \cos a \phi}$. Then, it is expected that $\varepsilon$ is much smaller than $\eta$ in our model.

The inflation would occur at the very flat region of the potential, i.e., $V_{\phi \phi} \approx 0$, and thus we approximate $V_{\phi \phi}$ as $V_{\phi \phi} \sim V_{\phi \phi \phi} \Delta \phi$ during the inflation. Then, we estimate the slow-roll parameter $\eta$,

$$
\begin{aligned}
|\eta| & \sim \frac{\left|V_{\phi \phi \phi}\right| \Delta \phi}{V} \\
& \sim \frac{A \delta^{3} a^{3} e^{-\delta \cos a \phi}|\sin (\delta \sin (a \phi)-4 a \phi+\theta)| \Delta \phi}{A V_{0}} \\
& \sim \delta^{2} a^{2} \sqrt{\varepsilon} \Delta \phi .
\end{aligned}
$$

Here, the phases of sine functions in $V_{\phi}$ and $V_{\phi \phi \phi}$ are different from each other, but we estimate that the values of sine functions are similar. That is, we can write

$$
(\Delta \phi)^{2} \sim \frac{\eta^{2}}{\delta^{4} a^{4} \varepsilon}
$$

By using the Lyth relation (3.6), we obtain

$$
r \sim 10^{-2} \frac{\eta^{2}}{\delta^{4} a^{4} \varepsilon}
$$

We have to realize $\eta \sim-0.01$, in order to derive $n_{s}=0.9655$, as mentioned above. Then, by use of $\eta \sim$ -0.01 and $r=16 \varepsilon$, the relation (3.12) leads to 

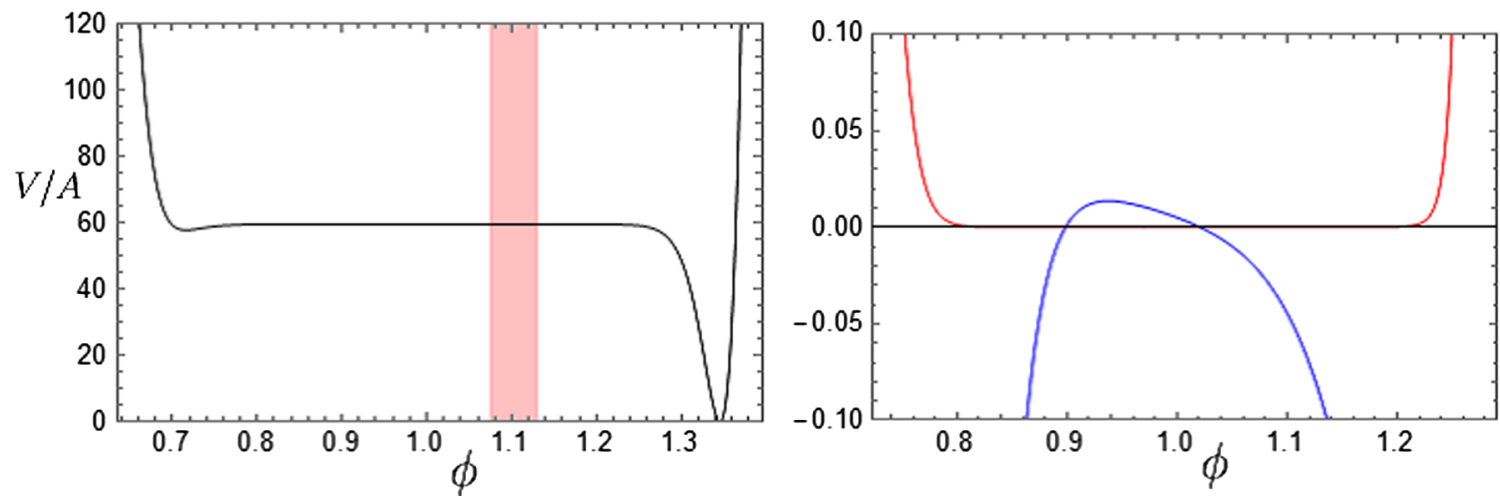

FIG. 1. The inflation potential with $\delta=8.0$. The red and blue curves correspond to $\varepsilon$ and $\eta$, respectively.
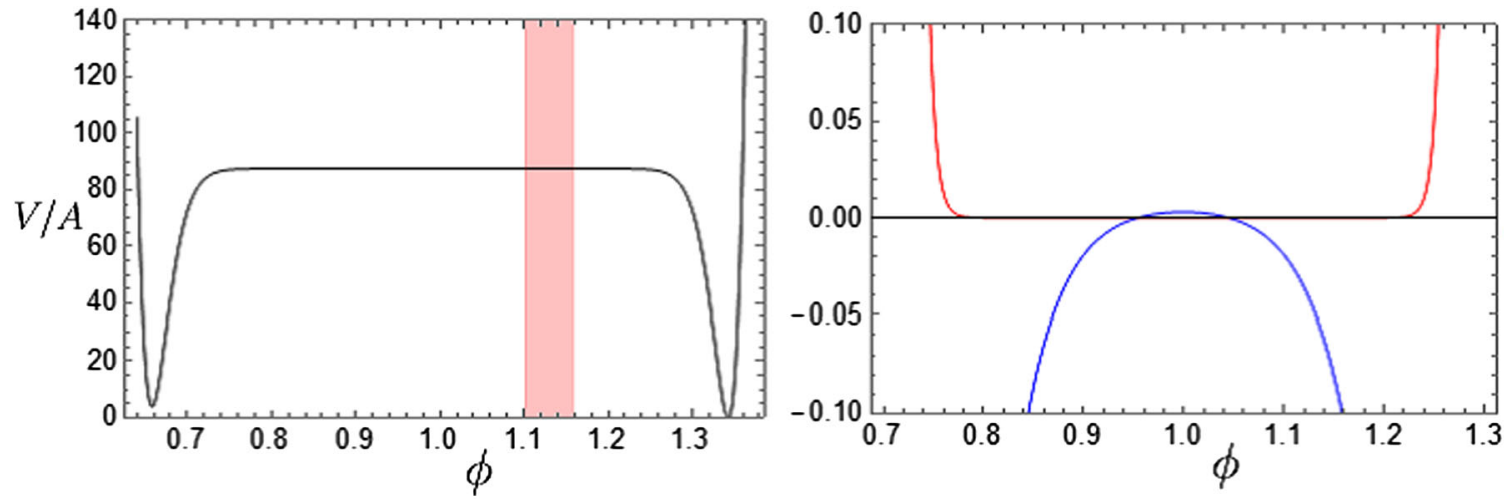

FIG. 2. The inflation potential with $\delta=9.0$. The red and blue curves correspond to $\varepsilon$ and $\eta$, respectively.

$$
\varepsilon^{2} \sim \frac{10^{-7}}{\delta^{4} a^{4}}
$$

For example, for $\delta=10, a=2 \pi$, we can estimate

$$
\varepsilon \sim 10^{-7}, \quad r=16 \varepsilon \sim 10^{-6} .
$$

From the relations among $P_{\xi}, \varepsilon$ and $V \approx A V_{0}$, we find

$$
V=A V_{0}=5.2 \varepsilon \times 10^{-7} \sim \frac{10^{-10}}{\delta^{2} a^{2}} .
$$

Then, the inflaton mass can be estimated as

$$
m_{\phi}^{2} \sim V_{\phi \phi} \sim A \delta^{2} a^{2} e^{-\delta_{\min }} \sim 10^{-10},
$$

where we used $V_{0} \sim e^{-\delta_{\min }}$. Note that the above inflaton mass does not depend explicitly on $\delta$ and $a$. We can estimate $m_{\phi}^{2} \sim 10^{-10}$, i.e., $m_{\phi} \sim 10^{13} \mathrm{GeV}$. Also, we estimate the running,

$\alpha_{s}=\frac{d n_{s}}{d \ln k} \sim-2 \xi=-2 \frac{V_{\phi} V_{\phi \phi \phi}}{V^{2}} \sim \varepsilon \eta(\delta a)^{2} \sim-10^{-3}$, which does not depend explicitly on $\delta$ and $a$. The running is negative and rather large. A similar value has been obtained in other axion inflation models $[9,14]$.

Finally, let us estimate the reheating temperature. The modulus field $T_{0}$ would appear in the gauge kinetic function of the visible sector through the instanton effect,

$$
f_{\text {vis }}=f_{0}+B_{\text {vis }} e^{-b_{\text {vis }} T_{0}} .
$$

Through this coupling, the inflaton-axion $\phi$ can decay into gauge bosons, and its decay rate can be estimated by

$$
\Gamma \sim \gamma^{2}\left(\frac{m_{\phi}}{10^{13} \mathrm{GeV}}\right)^{3} \mathrm{GeV},
$$

where $\gamma=B_{\text {vis }} b_{\text {vis }} e^{-b_{\text {vis }} \tau_{0}}$. When this process is dominant in the decay of $\phi$, the reheating temperature could be estimated as

$T_{r}=\left(\frac{\pi^{2} g_{*}}{90}\right)^{-1 / 4} \sqrt{\Gamma M_{\mathrm{Pl}}} \sim 10^{9} \gamma\left(\frac{m_{\phi}}{10^{13} \mathrm{GeV}}\right)^{3 / 2} \mathrm{GeV}$,

with the effective degrees of freedom $g_{*}=106.75$. Depending on $\gamma$, the reheating temperature becomes lower. 


\section{CONCLUSION}

We have investigated the axion potential generated by polyinstanton effects. In superstring theory, there are $h_{1,1}$ Kähler moduli and they remain massless at tree level. The $\alpha^{\prime}$ correction and one-loop correction in the Kähler potential can generate masses for the Kähler moduli, but the potential of axions would keep flat directions. The axions can be stabilized by nonperturbative effects and their masses could be lighter than other moduli. Thus, the natural inflation with and without modulations would be a good candidate for string-derived inflation models. Recently, another type of nonperturbative effect, i.e., the so-called polyinstanton effect, was studied. Thus, it is interesting to study the axion potential due to the polyinstanton effects and the possibility of the cosmic inflation.

We have derived a new axion inflation model generated by polyinstanton effects. The form of the polyinstanton potential is specific. Since the derivatives of the potential are exponentially suppressed by nonperturbative effects, the potential can have a flat region without severe finetuning of parameters. We have shown that the axion inflation is possible with our new potential, and that corresponds to the small-field inflation scenario. We numerically analyze its potential and find some parameter sets realizing the inflation. The magnitude of the polyinstanton effects is represented by the parameter $\delta$ and the large value of $\delta$ is favored to realize the inflation. Most of the observables depend on the parameters, and we have shown their dependencies on $\delta a$. For example, the tensor-to-scalar ratio is proportional to $\delta^{-4} a^{-4}$, and it becomes smaller as $\delta a$ becomes larger. On the other hand, the inflaton mass is independent of $\delta$ and $a$ and is of $\mathcal{O}\left(10^{13}\right) \mathrm{GeV}$. Also, the running $\alpha_{s}$ is independent of $\delta$ and $a$. Its value is negative and rather large, $\alpha_{s} \sim-10^{-3}$. A similar value has been obtained in other axion inflation models $[9,14]$. This behavior might be a generic feature in a certain class of the axion inflation models.

In this paper, we have assumed the existence of polyinstanton effects, but more precisely, it depends on the geometry of the compact space. For more concrete discussions, one should define the geometry giving rise to polyinstanton effects. In addition, the value of $\delta$ is significant for the polyaxion inflation, although we treat it as a free parameter. Moreover, the polyinstanton effects in the gauge kinetic function of the visible sector would be important, because it affects the decay of the axion and the reheating processes. In principle, the prefactor can be calculated as a one-loop partition function. It may be interesting to investigate it explicitly in concrete models. That is beyond our scope, and we will study it elsewhere.

\section{ACKNOWLEDGMENTS}

T. K. is supported in part by the Grant-in-Aid for Scientific Research Grants No. 26247042 and No. 17H05395 from the Ministry of Education, Culture, Sports, Science, and Technology in Japan.
[1] D. Baumann and L. McAllister, arXiv:1404.2601 [Cambridge University Press (to be published)].

[2] K. Freese, J. A. Frieman, and A. V. Olinto, Phys. Rev. Lett. 65, 3233 (1990).

[3] E. Silverstein and A. Westphal, Phys. Rev. D 78, 106003 (2008); L. McAllister, E. Silverstein, and A. Westphal, Phys. Rev. D 82, 046003 (2010).

[4] N. Kaloper and L. Sorbo, Phys. Rev. Lett. 102, 121301 (2009).

[5] J. E. Kim, H. P. Nilles, and M. Peloso, J. Cosmol. Astropart. Phys. 01 (2005) 005.

[6] H. Abe, T. Kobayashi, and H. Otsuka, Prog. Theor. Exp. Phys. 2015, 63E02.

[7] H. Abe, T. Kobayashi, and H. Otsuka, J. High Energy Phys. 04 (2015) 160.

[8] M. Czerny and F. Takahashi, Phys. Lett. B 733, 241 (2014); M. Czerny, T. Higaki, and F. Takahashi, J. High Energy Phys. 05 (2014) 144.

[9] T. Kobayashi, A. Oikawa, N. Omoto, H. Otsuka, and I. Saga, Phys. Rev. D 95, 063514 (2017).
[10] T. Kobayashi, O. Seto, and Y. Yamaguchi, Prog. Theor. Exp. Phys. 2014, 103E01 (2014); T. Higaki, T. Kobayashi, O. Seto, and Y. Yamaguchi, J. Cosmol. Astropart. Phys. 10 (2014) 025.

[11] T. Kobayashi, D. Nitta, and Y. Urakawa, J. Cosmol. Astropart. Phys. 08 (2016) 014.

[12] T. Higaki and Y. Tatsuta, arXiv:1611.00808.

[13] T. Kobayashi, A. Oikawa, and H. Otsuka, Phys. Rev. D 93, 083508 (2016).

[14] S. Parameswaran, G. Tasinato, and I. Zavala, J. Cosmol. Astropart. Phys. 04 (2016) 008.

[15] K. Kadota, T. Kobayashi, A. Oikawa, N. Omoto, H. Otsuka, and T. H. Tatsuishi, J. Cosmol. Astropart. Phys. 10 (2016) 013.

[16] K. Akita, T. Kobayashi, and H. Otsuka, J. Cosmol. Astropart. Phys. 04 (2017) 042.

[17] R. Blumenhagen and M. Schmidt-Sommerfeld, J. High Energy Phys. 07 (2008) 027.

[18] R. Blumenhagen, X. Gao, T. Rahn, and P. Shukla, J. High Energy Phys. 06 (2012) 162. 
[19] M. Cicoli, F. G. Pedro, and G. Tasinato, J. Cosmol. Astropart. Phys. 12 (2011) 022.

[20] M. Cicoli, C. P. Burgess, and F. Quevedo, J. High Energy Phys. 10 (2011) 119.

[21] R. Blumenhagen, X. Gao, T. Rahn, and P. Shukla, J. High Energy Phys. 11 (2012) 101.

[22] X. Gao and P. Shukla, J. High Energy Phys. 03 (2013) 061.

[23] X. Gao, T. Li, and P. Shukla, J. Cosmol. Astropart. Phys. 10 (2014) 008.

[24] M. Cvetic, R. Richter, and T. Weigand, Phys. Rev. D 76, 086002 (2007).

[25] R. Blumenhagen, M. Cvetic, S. Kachru, and T. Weigand, Annu. Rev. Nucl. Part. Sci. 59, 269 (2009).

[26] S. B. Giddings, S. Kachru, and J. Polchinski, Phys. Rev. D 66, 106006 (2002).
[27] S. Kachru, R. Kallosh, A. D. Linde, and S. P. Trivedi, Phys. Rev. D 68, 046005 (2003).

[28] T. Kobayashi, N. Omoto, H. Otsuka, and T. H. Tatsuishi, arXiv: 1704.04875.

[29] O. Lebedev, H. P. Nilles, and M. Ratz, Phys. Lett. B 636, 126 (2006); E. Dudas, C. Papineau, and S. Pokorski, J. High Energy Phys. 02 (2007) 028; H. Abe, T. Higaki, T. Kobayashi, and Y. Omura, Phys. Rev. D 75, 025019 (2007); R. Kallosh and A. D. Linde, J. High Energy Phys. 02 (2007) 002; H. Abe, T. Higaki, and T. Kobayashi, Phys. Rev. D 76, 105003 (2007).

[30] P. A. R. Ade et al. (Planck Collaboration), Astron. Astrophys. 571, A22 (2014).

[31] P. A. R. Ade et al. (Planck Collaboration), Astron. Astrophys. 594, A20 (2016).

[32] D. H. Lyth, Phys. Rev. Lett. 78, 1861 (1997). 